\title{
Title: Diagnosing Alzheimer's Disease from Circulating Blood Leukocytes Using a Fluorescent Amyloid Probe
}

Stefanie A G Black 12 , Anastasiia A Stepanchuk 1, George W Templeton 2, Yda Hernandez 3, Tomoko Ota 1, Shyamosree Roychoudhury 1, Eric E Smith 1, Philip A Barber 14 5, Zahinoor Ismail 165 7, Karyn Fischer 1, Angela Zwiers 1, Marc J Poulin 1 73, Kaj Blennow 8 9, Henrik Zetterberg 8910 11, Peter K Stys 1, Shigeki Tsutsui 12

1Hotchkiss Brain Institute, Department of Clinical Neurosciences, Cumming School of Medicine, University of Calgary, Calgary AB, Canada.

2Amira Medical Technologies Inc., Calgary, AB, Canada.

3Department of Physiology and Pharmacology, Libin Cardiovascular Institute of Alberta, Faculty of Kinesiology, University of Calgary, Calgary AB, Canada.

4Calgary Stroke Program, Seaman Family MR Center, Departments of Clinical Neurosciences and Radiology, Foothills Medical Centre, Calgary AB, Canada. 5Department of Community Health Sciences, University of Calgary, Calgary AB, Canada.

6Department of Psychiatry, and the Mathison Centre for Mental Health Research \& Education, Cumming School of Medicine, University of Calgary, Calgary, Alberta, Canada.

7O'Brien Institute of Public Health, University of Calgary, Calgary AB, Canada. 8Clinical Neurochemistry Laboratory, Sahlgrenska University Hospital, Gothenburg, Sweden.

9Department of Psychiatry and Neurochemistry, Institute of Neuroscience and Physiology, Sahlgrenska Academy at University of Gothenburg, Gothenburg, Sweden. 10Department of Neurodegenerative Disease, University College London Queen Square Institute of Neurology, London, UK.

11UK Dementia Research Institute, University College London, London, UK. 


\section{Abstract}

Background: Toxic amyloid- $\beta$ (A $\beta$ ) peptides aggregate into higher molecular weight assemblies and accumulate not only in the extracellular space, but also in the walls of blood vessels in the brain, increasing their permeability, and promoting immune cell migration and activation. Given the prominent role of the immune system, phagocytic blood cells may contact pathological brain materials.

Objective: To develop a novel method for early Alzheimer's disease (AD) detection, we used blood leukocytes, that could act as "sentinels" after trafficking through the brain microvasculature, to detect pathological amyloid by labelling with a conformationallysensitive fluorescent amyloid probe and imaging with confocal spectral microscopy.

Methods: Formalin-fixed peripheral blood mononuclear cells (PBMCs) from cognitively healthy control $(\mathrm{HC})$ subjects, mild cognitive impairment $(\mathrm{MCl})$ and $A D$ patients were stained with the fluorescent amyloid probe K114, and imaged. Results were validated against cerebrospinal fluid (CSF) biomarkers and clinical diagnosis.

Results: K114-labeled leukocytes exhibited distinctive fluorescent spectral signatures in $\mathrm{MCl} / \mathrm{AD}$ subjects. Comparing subjects with single CSF biomarker-positive $\mathrm{AD} / \mathrm{MCI}$ to negative controls, our technique yielded modest AUCs, which improved to the 0.90 range when only $\mathrm{MCl}$ subjects were included in order to measure performance in an early disease state. Combining CSF A 42 and t-Tau metrics further improved the AUC to 0.93.

Conclusion: Our method holds promise for sensitive detection of AD-related protein misfolding in circulating leukocytes, particularly in the early stages of disease.

Keywords: Alzheimer's disease; $A \beta 42$; cerebrospinal fluid; conformationally-sensitive amyloid probes; p-Tau; peripheral blood mononuclear cells; spectral confocal microscopy; t-Tau. 
Alzheimer's disease (AD) is the most common cause of dementia throughout the world with nearly 60 million people affected (www.alzint.org). Despite much effort, there is neither a cure for AD nor a non-invasive clinical test to reliably establish the diagnosis with certainty. Given the failure of almost all therapeutic trials with anti-amyloid drug candidates (PMID: 29365294, 29914022), it is now generally accepted that any future therapy will only be potentially effective if started as early as possible in the course of the disease, possibly even in the clinically pre-symptomatic stage as a preventative measure. Therefore, it will be important to devise methods to detect disease early and predict cognitive decline based not on clinical status alone, but on the use of biomarkers in combination with clinical criteria, which is the most accurate predictor of future cognitive impairment due to AD (PMID: 24849862, 29653606). Thus, development of an inexpensive, non-invasive, rapid test for $A D$ is of utmost importance as the population ages and AD prevalence is predicted to reach 82 million by 2030 (www.alzint.org).

This age-related progressive neurodegenerative disorder is pathologically characterized by $\beta$-amyloid $(A \beta)$-containing amyloid plaques, neurofibrillary tangles, and synapse loss in the brain (PMID: 11274343). Evidence indicates that pathological hallmarks of AD i.e., misfolding and aggregation of cellular proteins, in particular $A \beta$ peptides and tau, together with impaired clearance and accumulation, may contribute to the pathogenesis of this disease. Toxic $A \beta$ peptides aggregate into higher molecular weight assemblies and accumulate not only in the extracellular space, but also in the walls of blood vessels in the brain (PMID: 11935076, 17430246), increasing their permeability (PMID: 19542618), and promoting immune cell migration and activation (PMID: 12831163). Macrophages/microglia ingest $A \beta$ and are key players for $A \beta$ clearance (PMID: 17222479, $18026096,16476660)$. Monocytes clear $A \beta$ accumulated around veins and are able to circulate back to the bloodstream (PMID: 24210819). Neutrophils also infiltrate into AD brain by virtue of blood-brain barrier (BBB) disruption (PMID: 15689955). Although lymphocytes are not phagocytic, intercellular protein transfer has been observed between these immune cells (termed trogocytosis) (reviewed in PMID: 17157208, 31481628). Interestingly, $A \beta$ is transported bidirectionally across the BBB, which suggests a dynamic equilibrium between brain and circulating $A \beta$ (PMID: 11438712, 9042383). Tau biomarkers have attracted attention because of this protein's prominent increase in preclinical AD patient cerebrospinal fluid (CSF), at a stage when only subtle changes of $A \beta$ pathology are detected (PMID: 33169916). Very recently, novel plasma $p$-Tau181 and p-Tau217 biomarkers are significantly increased in the preclinical stage of AD (PMID: 33169916). Moreover, tau phosphorylation state changes are associated with neurodegenerative and clinical markers of disease, as early as the initial increases in aggregated $A \beta$ begin (PMID: 32161412). Thus, all cellular elements in the blood could act as "sentinels", with a "memory" of their experience after trafficking through the brain and its microvasculature, where they encounter $A \beta$ and/or fibrous tau assemblies in abnormal amounts and, more importantly, in pathological conformations.

The misfolding and aggregation of $A \beta$ from its soluble physiological state into highly ordered fibrillar assemblies is associated with increased $\beta$-sheet content (PMID: 20190788). This pathological $\beta$-sheet-rich amyloid exhibits high-affinity binding to the classic amyloid probe Congo Red, leading to the term "congophilic" diseases. Conformationally-sensitive amyloid probes, such as luminescent conjugated 
oligothiophenes and Congo Red derivatives, are effective for detecting pathological amyloids in tissue sections, such as $A \beta$ fibrils, oligomers and tau tangles (refs: PMID: 12950445, Aslund2009, Nilsson2005, Hammarstrom2010). We reasoned that peripheral blood leukocytes that ingest pathological amyloid fragments as they traverse the brain vasculature (add refs to support this e.g. http://dx.doi.org/10.1016/i.celrep.2013.10.010) could be interrogated using spectral analysis of fluorescent amyloid probes, thereby reporting the degree of amyloid accumulation in the brain. To this end, we developed a blood cell-based technique for AD diagnosis using spectral microscopy of peripheral blood mononuclear cells (PBMCs) labeled with the Congo Red derivative K114 [(trans,trans)-1-bromo-2,5-bis-(4-hydroxy)styrylbenzene].

\section{Methods}

\section{Human $A D$ and control brains}

Human brain samples from subjects that were given the diagnosis of AD with Braak stage 5 or 6 , and samples from cognitively-normal subjects with Braak stage 0 or 1 were obtained from British AD bank.

\section{Human subjects and PBMC sample collection}

The study included a total of 90 subjects including 49 patients with $A D, 19$ patients with $\mathrm{MCl}$, and 22 healthy controls $(\mathrm{HC})$ diagnosed by standard clinical criteria?. All subjects underwent cognitive testing (need details from the clinical folks below), lumbar puncture for the core AD CSF biomarkers and blood collection for staining and imaging of PBMCs. Blood samples were collected into EDTA-containing tubes and processed to isolate PBMCs by gradient purification using Ficoll-Paque (GE Healthcare). Isolated cells were fixed in $10 \%$ neutral-buffered formalin (VWR) and stored at $4^{\circ} \mathrm{C}$. Formalinfixed human PBMCs were smeared and dried on Superfrost Plus glass slides. Fixed PBMC smears were used for immunofluorescence and amyloid probe staining.

\section{CSF analysis}

CSF levels of A $A 42$, total tau (tTau) and phospho-tau (pTau) were analyzed using the Innotest ELISA methods (Fujirebio, Ghent, Belgium), while CSF A 440/42 ratio [(Aß42/ $A \beta 40) \times 10]$ was determined using the V-PLEX A $\beta$ Peptide Panel 1 (6E10) Kit (Meso Scale Discovery, Rockville, MD). The analyses were performed by board-certified laboratory technicians using established procedures for quality control and run acceptance. CSF ELISA-negative $\left(\mathrm{CSF}^{-}\right)$or -positive $\left(\mathrm{CSF}^{+}\right)$subjects (Table 1) were selected for validation.

\section{Spectral detection of amyloid}


Conformationally sensitive fluorescent probes that specifically bind amyloid have been widely used for the investigation of protein aggregation. Some of these probes have the additional property of detecting soluble oligomeric species and alter their emission spectra depending on the protein aggregate to which they bind, allowing them to identify minute deposits or subtle changes in conformation. K114 was selected as a best conformationally-sensitive fluorescent probe for our assay based on our preliminary studies with several candidates. PBMCs were stained with $300 \mu \mathrm{M} \mathrm{K} 114$ ((trans, trans)1-bromo-2,5-bis(4-hydroxystyryl)benzene, Tocris Bioscience) ) at $\mathrm{pH} 10.5$ in $0.1 \mathrm{M}$ $\mathrm{NaHCO}_{3}-\mathrm{NaOH}$ buffer containing 20\% DMSO and 50\% Fluoromount/Plus mounting medium. Stained PBMC smears were imaged with a Nikon C2si 32 channel spectral confocal microscope using $405 \mathrm{~nm}$ excitation. Emission spectra were collected from 400 to $720 \mathrm{~nm}$ in $10 \mathrm{~nm}$ increments. Images were manually masked to select PBMCs for analysis; contaminating RBCs, platelets, large cellular aggregates and non-cellular debris were excluded. Spectral images were analyzed using custom software written by PKS (ImageTrak: https://www.ucalgary.ca/styslab/imagetrak).

\section{Results}

\section{Fluorescence spectroscopy of K114 in human AD brain}

Pathologically confirmed human brain samples were used to define the spectral characteristics of K114-labeled amyloid as a typical K114-positive AD plaque spectrum. Our optimized fluorescent amyloid probe staining method with K114 showed morphologically very clear senile plaques with red-shifted emission spectra in the human brains (Fig. 1A). "Truecolour" images, calculated from the spectral confocal micrographs to approximate the actual colour that the naked eye would perceive if viewing the sample through the microscope oculars, showed typical human senile plaques brightly stained with K114 (Fig. 1A, left panels). Each pixel in the spectral image contains 32 channels of spectrally resolved data ranging from 400 to $720 \mathrm{~nm}$ with $10 \mathrm{~nm}$ bins. Two extreme fluorescence spectra, from healthy control $(\mathrm{HC})$ brain background (very blue-shifted nonpathological background (violet trace)) and AD plaque (very red-shifted plaque core (red trace)) spectra are extracted from spectral images of human subjects (Figure 1B). The spectral pseudocolour image shows an $A D$ brain plaque with a large spectral shift between core and rim (Fig. 1A, right panels). The deposition of $\beta$-sheet-rich amyloid in the plaques caused an intensity increase and a marked red-shift of emitted fluorescence. This shift exhibited notable spectral variation even within a single plaque, which is indicative of intraplaque heterogeneity of amyloid structure with various degrees of amyloid aggregation varying continuously from plaque periphery to plaque core. 
Spectral scatter analysis (SSA)

In order to analyze and quantitate these information-rich spectral micrographs, a novel method of analyzing spectral image data was developed, which we termed Spectral Scatter Analysis (SSA), allowing for high sensitivity and specificity. Briefly, spectral image data were divided into square kernels $\left(0.86 \mu \mathrm{m}^{2}\right)$, from which an overall fluorescence intensity and averaged spectrum are calculated, together with a continuous index based on spectral shape, representing the extent of amyloid pathology in each kernel (index 0 is normal, index 1 is mature pathological amyloid). Pixel intensities were plotted against spectral shape (computed as a single scalar index, with higher values indicating a more red-shifted spectrum) in a 2D scatterplot, from which an overall score is calculated representing an "amyloid load" based on a specific subpopulation of kernels as shown in the green $\mathrm{ROI}$ of Fig. $1 \mathrm{C}$. The main cluster in both $\mathrm{HC}$ and $\mathrm{AD}$ graphs represents the majority of background pixels in the brain parenchyma. Notably, the AD samples exhibited a unique additional collection of points extending upwards (higher intensity) and to the right (redder spectra, higher index), representing the plaques seen only in the AD brains. Selecting the AD-specific pixels (green rectangle in Fig. 1C), and applying the same ROI for all samples, allowed us to calculate objective statistics representing the "amyloid load" as brain plaque amyloid (brain plaque amyloid = index $\times$ intensity) in each sample. These results are plotted in Fig. 1D, indicating a highly significant difference between HC and AD as expected, mainly driven by plaque deposition in the latter.

\section{Fluorescence spectroscopy of K114 in human PBMCs}

The previous examples from brain illustrate our approach for quantitating amyloid load in biological samples. We hypothesized that circulating leukocytes could act as "sentinels", reflecting the amyloid status of diseased brain. To test this idea, we examined K114labeled PBMCs from $\mathrm{HC}$ and MCl/AD patient blood to see if amyloid-like spectral shifts could be detected. In contrast to brain images, where bright plaques are easily seen, K114-labeled human PBMC truecolour images from HC vs AD patients are not distinguishable visually (Fig. 2A, left panels). Fig. $2 B$ illustrates that in PBMCs, the spectral differences between $\mathrm{HC}$ and $\mathrm{AD}$ samples are less pronounced compared to brain, accounting for the similarity in the truecolour image appearance. Importantly, however, although spectral differences were more subtle, PBMC spectra from AD subjects also exhibited a red-shift, as in brain, which is a characteristic of this probe when binding to $A \beta$ (Levine 2005). Pseudocolour images, designed to emphasize subtle spectral shifts, revealed clear differences between $H C$ and $A D$, quantitatively shown by SSA analysis in Fig. 2C. Although much more subtle, similarly to brain, PBMCs from AD subjects tended to exhibit red-shifted spectra (right-shifted distributions along the horizontal index axis) whose intensities were higher than corresponding pixels from control subjects (green ROI in Fig. $2 \mathrm{C}$ ). However, although the patterns were similar to those in brain, the extent of spectral red-shift and intensity increase was less pronounced in PBMCs from AD 
compared to $\mathrm{HC}$ subjects. This likely mirrors differences in quantity and state of amyloid from the two types of sample.

\section{PBMC spectral analysis and CSF biomarkers}

To determine how well the spectral amyloid assay could detect disease, we analyzed the PBMC score by using the enhanced quantitative method based on our preliminary study and compared with CSF biomarkers. Subject characteristics, PBMC scores and CSF biomarkers are reported in Table 1. Mean ages did not differ between the groups. Established AD CSF biomarkers showed characteristic profiles of reduced $A \beta 42$ and increased t-Tau and p-Tau in the clinical $\mathrm{MCl}$ and $\mathrm{AD}$ subjects, whereas the $\mathrm{HC}$ group had the expected higher levels of $A \beta 42$ and low levels of t-Tau and $p$-Tau as previously reported (PMID: 27068280).

As an initial gauge of the performance of our fluorescent method, we plotted PBMC scores against single CSF biomarker status based on previously published cut off values (Table 1 ), independent of clinical diagnosis (all 90 subjects included). Although PBMC scores were significantly different between control and disease cohorts for each of the CSF AB42 (Fig. 3A), t-Tau (Fig. 3B) and p-Tau (Fig. 3C) single biomarker grouping, the receiver operating curves resulted in modest AUCs (0.71 - 0.74 range; Fig. 3D). Plots of continuous sensitivities, specificities and Youden indexes as a function of various cutoffs were shown in Fig. $3 E(A \beta 42)$ and $F(t-T a u)$. All three comparisons grouped by CSF A 342 , $\mathrm{t}$-Tau and $\mathrm{p}$-Tau showed very similar diagnostic accuracy (dashed line, used as a baseline in later analyses). Interestingly, near-optimal Youden indexes are found for a narrow cutoff in the A 342 -based group (Fig. 3E) compared to a relatively wider range of peaks in both t-Tau- (Fig. 3F) and p-Tau- (data not shown) based groups. The limitation of this analysis includes the potential uncertainty of clinical diagnosis of AD-related cognitive impairment particularly in the earlier stages of $\mathrm{MCl}$, nor do we know a priori whether our assay mirrors clinical dementia, brain amyloid status independent of functional cognitive decline, or a combination of both. To shed further light on this question, we next analyzed the PBMC scores from subjects who were CSF biomarker-negative, clinically $\mathrm{HC}$ and those who were biomarker-positive, clinically $\mathrm{MCl}$, as $\mathrm{MCl}$ subjects are considered predementia and later often progress to $A D$ (ref)-(Fig. 4). Comparing CSF biomarker-negative to CSF biomarker-positive subjects within this restricted cohort (AD subjects excluded) yielded statistically significant differences in the PBMC score once again (Fig. 4A-C), and with AUCs improving markedly to the high 0.8 range; notably, classification by clinical HC vs. $\mathrm{MCl}$ and by CSF p-Tau biomarkers yielded a PBMC score AUC approaching 0.9 (Fig. 4D compared to 3D) as well as a Youden index improving from 0.45 (Fig. $3 \mathrm{E}$ and F) to 0.72 (Fig. 4E and F).

Combining CSF markers often improves diagnostic accuracy (PMID: 22078172), therefore, we next evaluated the PBMC score grouped based on combination of CSF A $\beta$ and tau. A recent study showed ratios of $A \beta 42 / A \beta 40$ and $A \beta 42 / t-T a u$ are very reliable and have high diagnostic accuracy (PMID: 31010420). Comparing either $A \beta 42 / A \beta 40-$ or $A \beta 42 / t-T a u-n e g a t i v e$ to $A \beta 42 / A \beta 40$ - or $A \beta 42 / t-T a u$-positive PBMC score groups with all 
$\mathrm{HC}, \mathrm{MCl}$ and $\mathrm{AD}$ subjects yielded statistically significant differences with much higher statistical significance for the A $342 / t-T a u$ comparison (Fig. 5B). However, when we analyzed the diagnostic accuracies for these two combined CSF biomarkers, the AUCs did not differ from the analyses with single CSF biomarkers (AB42, t-Tau or $p$-Tau alone) (Fig. 5D compared to 3D). The prevailing view of $A D$ pathogenesis has been that initial $A \beta$ precipitation in the disease process triggers a detrimental cascade involving tau pathology and neurodegeneration for many years. However, accumulating evidence now suggests that both $A \beta$ and Tau pathologies have synergistic effects (PMID: 32778792), and thus combining these two biomarkers could improve diagnostic reliability. Therefore, we calculated combined $A \beta 42$ and $t-T a u$ metrics based on the hypothesis that $A \beta$ and Tau synergistically represent a patient's disease severity (Fig. 5C; 550/A $342+\mathrm{t}-\mathrm{Tau} / 400$ ). Interestingly, the PBMC score grouped by this method alone, without considering clinical status showed markedly higher AUC (Fig. 5D) and Youden index (Fig. 5F) compared to

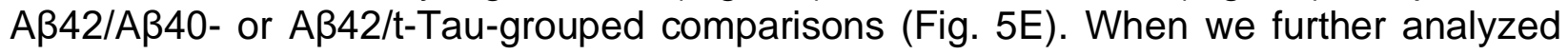
the PBMC score using combined CSF biomarker-negative and -positive groups (using the above CSF metrics) only in clinically established $\mathrm{HC}$ and $\mathrm{MCl}$ subjects (Fig. 6), comparing combined $A \beta 42 \& t-T a u$-negative to -positive within $\mathrm{HC}$ and $\mathrm{MCl}$ subjects (AD subjects excluded) showed the highest group separation (Fig. 6D and F).

\section{Discussion}

Whereas analyzing post-mortem brain is the de facto standard for AD diagnosis, there is no non-invasive clinical test to reliably establish the diagnosis with certainty. Biological fluid testing, including CSF and other developing blood-based analyses, rely on quantification of the monomeric units of amyloid, either $A \beta$ or Tau, and on their phosphorylation state. While monomer status is strongly correlated with amyloid state, it is not a direct measurement of amyloid status. In this study, we introduce a novel blood $A D$ biomarker based on fluorescence microspectroscopy of the amyloid-binding molecular probe $\mathrm{K} 114$ after staining of circulating blood mononuclear cells (PBMCs). These circulating phagocytic cells are known to ingest amyloid while traversing the brain microvasculature. The $A D$ brain parenchyma accumulates two main amyloid species: misfolded/aggregated $A \beta$ and Tau. Interestingly, when we measured total levels of these proteins in PBMCs using conventional immunocytochemical methods that generally do not distinguish between normally folded vs. misfolded/aggregated proteins, we did not detect quantitative differences between controls and MCl/AD subjects (Fig. X, upcoming $\mathrm{IHC}$ ). We reasoned that it may not be the total amounts of $A \beta$ and Tau proteins contained in these cells, but rather, amounts of misfolded, aggregated $\beta$-sheet-rich species that could detect $A D$ brain pathology. A number of fluorescent probes have been developed that bind to and are relatively specific for $\beta$-sheet amyloids. Congo Red and Thioflavin- $T$ are the best known, but a variety of other probes with very dissimilar structures have also been reported. We chose $\mathrm{K} 114$ because it is a bright, high-affinity probe for a variety of amyloids, including aggregated $A \beta$ and Tau. Importantly, K114 undergoes a shift in its emission spectrum when binding amyloids, which allows detection by spectral shape, rather than absolute fluorescence intensity alone, which is very difficult to control. Staining PBMCs with this spectrally variable probe allowed us to compute a score, based on relative intensity and spectral shape. The initial analysis using this PBMC fluorescence 
score to identify $\mathrm{MCl}$ and/or $\mathrm{AD}$ compared to $\mathrm{HC}$ was very encouraging and showed similar diagnostic accuracy of CSF t-Tau and p-Tau, but lower than AB42 and combined CSF biomarkers (Table 1), which suggests our novel assay may correlate better with CSF Tau markers than $A \beta$ markers.

The trend of better correlation between our novel blood AD test and CSF Tau markers was observed throughout this study, even when subjects were classified based on single CSF biomarker positivity, independent of clinical diagnosis. Interestingly, this trend was more prominent when we compared only the clinically normal subjects $(\mathrm{HC})$ and subjects with $\mathrm{MCl}$, including possibly pre-clinical AD but not symptomatic AD subjects (Table 2 ). This suggests our PBMC method may be better at detecting early stages of $A D$ (in the $\mathrm{MCl}, \mathrm{MBI}$ (or even pre-clinical) rather than already established late-stage AD. This confers an important advantage since recent data suggest that disease-modifying therapies are likely to be most effective when started early in the disease (refs).

The highest diagnostic accuracy with single CSF biomarkers was shown when the subjects were classified by $\mathrm{p}$-Tau within the restricted $(\mathrm{HC}$ and $\mathrm{MCl})$ cohorts. Our results are consistent with recent studies that reported increased plasma $p$-Tau181 levels in the preclinical stage of AD (PMID: 33169916), and strong association of tau phosphorylation state changes with neurodegenerative and clinical markers of disease, occurring as early as the initial increases in aggregated A $\beta$ (PMID: 32161412).

Grouping the PBMC score based on the combination of both A $\beta$ and Tau CSF biomarkers improved the diagnostic accuracy overall compared to the grouping by any single CSF biomarker (Fig. 6). We attempted three different combinations: Aß42/A $\beta 40, A \beta 42 / t-T a u$ and $A \beta 42 \& t-T a u$. The highest diagnostic accuracy was found when subjects were classified by combined $A \beta 42$ \& t-Tau metrics based on the hypothesis that $A \beta$ and Tau synergistically reflect disease severity. This may represent unique characteristics of our novel spectroscopy blood AD detection method that are able to report overall amyloid load, including both $A \beta$ and Tau, which is suggested by the best results from a combined $A \beta 42$ \& $t$-Tau metric (Fig. 6C, D and F).

Together, these results indicate that our method is capable of sensitive and potentially AD-specific detection of protein misfolding/aggregation in circulating PBMCs. These data also support our hypothesis that circulating leukocytes act as "sentinels" with the potential ability to mirror brain amyloid status in a semi-quantitative manner.

\section{REFERENCES}

[1]

Honig LS, Vellas B, Woodward M, Boada M , Bullock R, Borrie M, Hager K, Andreasen N, Scarpini E , Liu-Seifert H, Case M, Dean RA, Hake A, Sundell K, Poole Hoffmann V , Carlson C, Khanna R, Mintun M , DeMattos R, Selzler KJ , Siemers E (2018) Trial of Solanezumab for mild dementia due to Alzheimer's disease. N Engl J Med 378, 321330. 
Salloway SP , Sperling R, Fox NC, Sabbagh MN , Honig LS, Porsteinsson AP, Rofael H, Ketter N , Wang D , Liu E , Carr S , Black RS , Brashear HR (2018) Long-term follow up of patients with mild-to-moderate Alzheimer's disease treated with Bapineuzumab in a phase iii, open-label, extension study. J Alzheimers Dis 64, 689-707.

[3]

Mortby ME, Black SE, Gauthier S, Miller D , Porsteinsson A, Smith EE , Ismail Z (2018) Dementia clinical trial implications of mild behavioral impairment. Int Psychogeriatr 30, $171-175$.

\section{[4]}

Dubois B , Feldman HH, Jacova C, Hampel H, Molinuevo JL, Blennow K, DeKosky ST, Gauthier S, Selkoe D, Bateman R, Cappa S, Crutch S, Engelborghs S, Frisoni GB , Fox NC , Galasko D, Habert MO , Jicha GA, Nordberg A, Pasquier F, Rabinovici G, Robert P , Rowe C , Salloway S, Sarazin M, Epelbaum S, de Souza LC, Vellas B, Visser PJ , Schneider L , Stern Y, Scheltens P , Cummings JL (2014) Advancing research diagnostic criteria for Alzheimer's disease: The IWG-2 criteria. Lancet Neurol 13, 614629.

[5]

Jack CR , Bennett DA, Blennow K, Carrillo MC, Dunn B, Haeberlein SB , Holtzman DM , Jagust W , Jessen F , Karlawish J , Liu E , Molinuevo JL, Montine T, Phelps C , Rankin KP , Rowe CC , Scheltens P , Siemers E , Snyder HM , Sperling R, Contributors (2018) NIA-AA Research Framework: Toward a biological definition of Alzheimer's disease. Alzheimers Dement 14, 535-562.

[6]

de la Torre JC (2002) Alzheimer disease as a vascular disorder: Nosological evidence. Stroke 33, 1152-1162.

[7]

Deane R , Zlokovic BV (2007) Role of the blood-brain barrier in the pathogenesis of Alzheimer's disease. Curr Alzheimer Res 4, 191-197.

[8]

Nagababu E, Usatyuk PV , Enika D , Natarajan V , Rifkind JM (2009) Vascular endothelial barrier dysfunction mediated by amyloid-beta proteins. J Alzheimers Dis 17, 845-854. 
[9]

Farkas IG , Czigner A, Farkas E, Dobó E, Soós K, Penke B, Endrész V , Mihály A (2003) Beta-amyloid peptide-induced blood-brain barrier disruption facilitates T-cell entry into the rat brain. Acta Histochem 105, 115-125.

[10]

Majumdar A, Chung H, Dolios G, Wang R, Asamoah N , Lobel P, Maxfield FR (2008) Degradation of fibrillar forms of Alzheimer's amyloid beta-peptide by macrophages. Neurobiol Aging 29, 707-715.

Mildner A, Schmidt $H$, Nitsche $M$, Merkler D , Hanisch UK, Mack M, Heikenwalder $M$, Brück W , Priller J , Prinz M (2007) Microglia in the adult brain arise from Ly6ChiCCR2+monocytes only under defined host conditions. Nat Neurosci 10, 1544-1553.

[12]

Simard AR, Soulet D , Gowing G , Julien JP , Rivest S (2006) Bone marrow-derived microglia play a critical role in restricting senile plaque formation in Alzheimer's disease. Neuron 49, 489-502.

[13]

Michaud JP , Bellavance MA , Préfontaine P , Rivest S (2013) Real-time in vivo imaging reveals the ability of monocytes to clear vascular amyloid beta. Cell Rep 5, 646-653.

[14]

Stamatovic SM , Shakui P, Keep RF , Moore BB , Kunkel SL, Van Rooijen N , Andjelkovic AV (2005) Monocyte chemoattractant protein-1 regulation of blood-brain barrier permeability. J Cereb Blood Flow Metab 25, 593-606.

[15]

Caumartin J , Lemaoult J , Carosella ED (2006) Intercellular exchanges of membrane patches (trogocytosis) highlight the next level of immune plasticity. Transpl Immunol 17, 20-22.

[16]

Dance A (2019) Core Concept: Cells nibble one another via the under-appreciated process of trogocytosis. Proc Natl Acad Sci U S A 116, 17608-17610. 
DeMattos RB , Bales KR , Cummins DJ, Dodart JC, Paul SM , Holtzman DM (2001) Peripheral anti-A beta antibody alters CNS and plasma $A$ beta clearance and decreases brain A beta burden in a mouse model of Alzheimer's disease. Proc Natl Acad Sci U S A $98,8850-8855$.

Shayo M , McLay RN , Kastin AJ , Banks WA (1997) The putative blood-brain barrier transporter for the beta-amyloid binding protein apolipoprotein $j$ is saturated at physiological concentrations. Life Sci 60, PL115-118.

Suárez-Calvet M , Karikari TK , Ashton NJ , LanteroRodríguez J , Milà-Alomà M , Gispert JD, Salvadó G, Minguillon C , Fauria K, Shekari M , Grau-Rivera O, Arenaza-Urquijo EM , Sala-Vila A , Sánchez-Benavides G, González-de-Echávarri JM , Kollmorgen G, Stoops E , Vanmechelen E, Zetterberg H, Blennow K, Molinuevo JL, ALFA S (2020) Novel tau biomarkers phosphorylated at T181, T217 or T231 rise inthe initial stages of the preclinical Alzheimer's continuum whenonly subtle changes in $A \beta$ pathology are detected. EMBOMol Med 12, e12921.

[20]

Palmqvist S, Janelidze S, Quiroz YT, Zetterberg H, Lopera F, Stomrud E , Su Y, Chen $Y$, Serrano GE , Leuzy A, Mattsson-Carlgren N , Strandberg O , Smith R, Villegas A, Sepulveda-Falla D, Chai X, Proctor NK, Beach TG, Blennow K, Dage JL, Reiman EM , Hansson O (2020) Discriminative accuracy of plasma phospho-tau217 for Alzheimer disease vs other neurodegenerative disorders. JAMA 324, 772-781.

[21]

Ashton NJ , Pascoal TA, Karikari TK, Benedet AL , Lantero-Rodriguez J , Brinkmalm G, Snellman A, Schöll M , Troakes C , Hye A, Gauthier S, Vanmechelen E, Zetterberg H, Rosa-Neto P , Blennow K (2021) Plasma p-tau231: A new biomarker for incipient Alzheimer's disease pathology. Acta Neuropathol 141, 709-724.

[22]

Barthélemy NR , Li Y, Joseph-Mathurin N , Gordon BA , Hassenstab J , Benzinger TLS, Buckles V , Fagan AM , Perrin RJ, Goate AM , Morris JC , Karch CM , Xiong C , Allegri R, Mendez PC, Berman SB, Ikeuchi T, Mori H, Shimada H, Shoji M , Suzuki K, Noble J , Farlow M , Chhatwal J , Graff-Radford NR , Salloway S , Schofield PR , Masters CL, Martins RN , O'Connor A, Fox NC, Levin J , Jucker M , Gabelle A, Lehmann S, Sato 
C , Bateman RJ , McDade E, Dominantly IAN (2020) A soluble phosphorylated tau signature links tau, amyloid and the evolution of stages of dominantly inherited Alzheimer's disease. Nat Med 26, 398-407.

[23]

Crystal AS , Giasson BI, Crowe A, Kung MP, Zhuang ZP , Trojanowski JQ, Lee VM (2003) A comparison of amyloid fibrillogenesis using the novel fluorescent compound K114. J Neurochem 86, 1359-1368.

Aslund A, Sigurdson CJ , Klingstedt T, Grathwohl S, Bolmont T, Dickstein DL, Glimsdal E, Prokop S , Lindgren M , Konradsson P, Holtzman DM , Hof PR , Heppner FL , Gandy $S$, Jucker M , Aguzzi A , Hammarström P , Nilsson KP (2009) Novel pentameric thiophene derivatives for in vitro and in vivo optical imaging of a plethora of protein aggregates in cerebral amyloidoses. ACS Chem Biol 4, 673-684.

[25]

Nilsson KP , Herland A , Hammarström P , Inganäs O (2005) Conjugated polyelectrolytes: Conformation-sensitive optical probes for detection of amyloid fibril formation. Biochemistry 44, 3718-3724.

[26]

Hammarström P, Simon R, Nyström S, Konradsson P, Aslund A, Nilsson KP (2010) A fluorescent pentameric thiophene derivative detects in vitro-formed prefibrillar protein aggregates. Biochemistry 49, 6838-6845.

[27]

Zaghi J , Goldenson B, Inayathullah M , Lossinsky AS, Masoumi A, Avagyan H, Mahanian M , Bernas M , Weinand M , Rosenthal MJ, Espinosa-Jeffrey A, de Vellis J , Teplow DB , Fiala M (2009) Alzheimer disease macrophages shuttle amyloid-beta from neurons to vessels, contributing to amyloid angiopathy. Acta Neuropathol 117, 111-124.

[28]

Stepanchuk AA, Heyne B , Stys PK (2021) Complex photophysical properties of K114 make for a versatile fluorescent probe for amyloid detection. ACS Chem Neurosci 12, 1273-1280. 
Huang V , Hogan DB , Ismail Z, Maxwell CJ , Smith EE , Callahan BL (2020) Evaluating the real-world representativeness of participants with mild cognitive impairment in Canadian research protocols: A comparison of the characteristics of a memory clinic patients and research samples. Can Geriatr J 23, 297-328.

[30]

Switzer AR , Cheema I, McCreary CR , Zwiers A, Charlton A, Alvarez-Veronesi A, Sekhon R , Zerna C , Stafford RB, Frayne R , Goodyear BG , Smith EE (2020) Cerebrovascular reactivity in cerebral amyloid angiopathy, Alzheimer disease, and mild cognitive impairment. Neurology 95, e1333-e1340.

Chertkow H, Borrie M, Whitehead V, Black SE , Feldman HH, Gauthier S, Hogan DB, Masellis M, McGilton K, Rockwood K, Tierney MC, Andrew M, Hsiung GR , Camicioli R, Smith EE , Fogarty J , Lindsay J, Best S, Evans A , Das S, Mohaddes Z, Pilon R , Poirier J , Phillips NA, MacNamara E, Dixon RA, Duchesne S, MacKenzie I, Rylett RJ (2019) The comprehensive assessment of neurodegeneration and dementia: Canadian Cohort Study. Can J Neurol Sci 46, 499-511.

[32]

LeVine $H$ (2005) Mechanism of $A$ beta(1-40) fibril-induced fluorescence of (trans, trans)1-bromo-2,5-bis(4-hydroxystyryl)benzene (K114). Biochemistry 44, 15937-15943.

Lee D , Kim SM , Kim HY , Kim Y (2019) Fluorescence chemicals to detect insoluble and soluble amyloid- $\beta$ aggregates. ACS Chem Neurosci 10, 2647-2657.

[34]

Olsson B , Lautner R, Andreasson U , Öhrfelt A, Portelius E, Bjerke M, Hölttä M , Rosén C , Olsson C , Strobel G, Wu E , Dakin K, Petzold M, Blennow K, Zetterberg H (2016) CSF andblood biomarkers for the diagnosis of Alzheimer's disease: Asystematic review and meta-analysis. Lancet Neurol 15, 673-684.

Miners JS, Kehoe PG, Love S, Zetterberg H , Blennow K (2019) CSF evidence of pericyte damage in Alzheimer's disease is associated with markers of blood-brain barrier dysfunction and disease pathology. Alzheimers Res Ther 11, 81. 
Smailovic U , Koenig T , Savitcheva I , Chiotis K, Nordberg A , Blennow K, Winblad B , Jelic V (2020) Regional disconnection in Alzheimer dementia and amyloid-positive mild cognitive impairment: Association between EEG functional connectivity and brain glucose metabolism. Brain Connect 10, 555-565.

Holtzman DM (2011) CSF biomarkers for Alzheimer's disease: Current utility and potential future use. Neurobiol Aging 32 Suppl 1, S4-9.

[38]

Hansson O , Lehmann S, Otto M , Zetterberg H , Lewczuk P (2019) Advantages and disadvantages of the use of the CSF Amyloid $\beta$ (A $\beta$ ) 42/40 ratio in the diagnosis of Alzheimer's disease. Alzheimers Res Ther 11, 34.

[39]

Ritchie C, Smailagic N, Noel-Storr AH, Ukoumunne O, Ladds EC, Martin S (2017) CSF tau and the CSF tau/ABeta ratio for the diagnosis of Alzheimer's disease dementia and other dementias in people with mild cognitive impairment (MCl). Cochrane Database Syst Rev 3, CD010803.

[40]

Busche MA , Hyman BT (2020) Synergy between amyloid- $\beta$ and tau in Alzheimer's disease. Nat Neurosci 23, 1183-1193.

Ismail Z, Smith EE, Geda Y, Sultzer D , Brodaty H , Smith G , Agüera-Ortiz L, Sweet R , Miller D , Lyketsos CG , ISTAART NSPIA (2016) Neuropsychiatric symptoms as early manifestations of emergent dementia: Provisional diagnostic criteria for mild behavioral impairment. Alzheimers Dement 12, 195-202.

[42]

Lussier FZ, Pascoal TA, Chamoun M , Therriault J , Tissot C , Savard M , Kang MS , Mathotaarachchi S, Benedet AL, Parsons M, Qureshi MNI, Thomas ÉM , Shin M , Dion LA , Massarweh G, Soucy JP , Tsai IH , Vitali P , Ismail Z, Rosa-Neto P, Gauthier S (2020) Mild behavioralimpairment is associated with $\beta$-amyloid but not tau orneurodegeneration in cognitively intact elderly individuals. Alzheimers Dement 16, $192-$ 199. 
Naude JP , Gill S, Hu S, McGirr A, Forkert ND , Monchi O, Stys PK, Smith EE, Ismail Z, Alzheimer's DNI (2020) Plasma neurofilament light: A marker of neurodegeneration in mild behavioral impairment. J Alzheimers Dis 76, 1017-1027.

[44]

Ismail Z , Agüera-Ortiz L , Brodaty H , Cieslak A, Cummings J , Fischer CE , Gauthier S, Geda YE , Herrmann N , Kanji J , Lanctôt KL, Miller DS , Mortby ME , Onyike CU , Rosenberg PB , Smith EE , Smith GS, Sultzer DL , Lyketsos C, NPS Professional Interest Area of thelnternational Society of to Advance Alzheimer's Research andTreatment (NPS-PIA of ISTAART (2017) The Mild Behavioral ImpairmentChecklist (MBI-C): A rating scale for neuropsychiatric symptoms inpre-dementia populations. J Alzheimers Dis 56, 929-938.

Ismail Z , McGirr A, Gill S , Hu S , Forkert ND , Smith EE (2021) Mild behavioral impairment and subjective cognitive decline predict cognitive and functional decline. $\mathrm{J}$ Alzheimers Dis 80, 459-469.

Cummings J , Lee G , Ritter A , Sabbagh M , Zhong K (2020) Alzheimer's disease drug development pipeline: 2020. Alzheimers Dement (N Y) 6, e12050.

Ritchie CW , Russ TC, Banerjee S, Barber B, Boaden A, Fox NC, Holmes C, Isaacs JD , Leroi I , Lovestone S, Norton M , O'Brien J , Pearson J , Perry R, Pickett J , Waldman AD , Wong WL , Rossor MN , Burns A (2017) The Edinburgh Consensus: Preparing for the advent of disease-modifying therapies for Alzheimer's disease. Alzheimers Res Ther 9,85 .

[48]

Yiannopoulou KG , Papageorgiou SG (2020) Current and future treatments in Alzheimer disease: An update. J Cent Nerv Syst Dis 12, 1179573520907397.

Tarawneh R , Holtzman DM (2012) The clinical problem of symptomatic Alzheimer disease and mild cognitive impairment. Cold Spring Harb Perspect Med 2, a006148. 
Albert MS, DeKosky ST, Dickson D , Dubois B , Feldman HH, Fox NC, Gamst A, Holtzman DM , Jagust WJ , Petersen RC, Snyder PJ , Carrillo MC , Thies B , Phelps CH (2011) The diagnosis of mild cognitive impairment due to Alzheimer's disease: Recommendations from the National Institute on Aging-Alzheimer's Association workgroups on diagnostic guidelines for Alzheimer's disease. Alzheimers Dement 7, 270 279. 